\title{
Fatigue of short fibre reinforced polymers: from material process to fatigue life of industrial components
}

\author{
Pablo Wilson ${ }^{1 *}$ and Peter Heyes ${ }^{2}$ \\ ${ }^{1}$ Renault S.A.S., 1 avenue du Golf, 78084 Guyancourt, France \\ ${ }^{2} \mathrm{HBM}$ Prenscia, Catcliffe, Rotherham S60 5WG, England
}

\begin{abstract}
For many years, SFRPs (short fibre reinforced polyamides) have been used in the automotive industry as a means to reduce vehicle weight. However, their complex anisotropic and heterogeneous microstructure requires sophisticated material characterisation and simulation. This study aims at presenting the simulation strategy adopted by an automotive company address these challenges. The manufacturing process is first simulated and correlated with tomography analysis. Then, based on the numerical microstructure, integrative simulation is used to analyse and predict mechanical behaviour of fatigue coupons and industrial parts. Lastly, two fatigue criteria based on strain energy density are presented and fatigue lives of coupons and industrial parts assessed.
\end{abstract}

\section{Introduction}

Faced with the environmental challenges of the 21st century, the automotive industry has had to adapt its material strategy. Indeed, current and future regulations are ever more demanding. To minimize the environmental footprint, vehicle weight has to be kept to a minimum to lower gas consumption, while engine performance has to be maximized to increase vehicle efficiency.

Plastics in general, and specifically short fibre reinforced plastics (SFRP) are often a good choice for more mechanically demanding applications: they combine a good specific mechanical strength, high production rate and low manufacturing costs, while providing the capability to be moulded in complex geometries.

However, designing such mechanical parts has generated new difficulties: the material microstructure caused by the fibre orientation produces material anisotropy. All other things being equal, the average fibre orientation can vastly modify mechanical characteristics [1].

In order to take into account the effect of the microstructure on the mechanical behaviour of SFRP, new lifetime assessment methods based on finite element analysis (FEA) have been developed. Firstly, the injection moulding is simulated and the fibre orientation in the part estimated. Secondly, integrative simulation is performed: this allows capture of the local anisotropy of the material and provides reliable mechanical fields (stress and strain). Lastly, since mechanical behaviour is here considered uncoupled with damage, the results can be post-processed and, using an appropriate fatigue criterion, the fatigue life assessed.

In this study, the different steps of this fatigue life assessment are presented on fatigue coupons and tested on industrial prototyped parts.

\section{Material and testing}

For confidentiality reasons, the results have been normalized. In order to remain intelligible, stress values have all been normalized by the same value $\sigma_{U T S}$ (ultimate tensile stress at $23^{\circ} \mathrm{C}, 50 \%$ relative humidity of the $0^{\circ}$ specimen).

\subsection{Coupons material and preparations}

The material used in this study is a polyamide reinforced with $50 \%$ weight glass fibres (hereafter referred to as PA66GF50). Further details on material are not disclosed for confidentiality reasons.

The fatigue test coupon geometry is presented in Figure 1, accordingly to ISO 527B standard.

These are machined from injected plates (see Figure 2). By using this method, different specimen "orientations" can be used. The " 0 " orientation hence corresponds to the specimen machined following the injection direction, while the " $90^{\circ}$ " one is taken perpendicular to it. The " $45^{\circ}$ " specimen is cut at a 45 degree angle from the injection direction.

Considering the sensitivity of the material to humidity (see [2]), the material is first conditioned at

*orresponding author: pablo.wilson@renault.com 
$50 \%$ relative humidity using an accelerated conditioning method described in the ISO 1110 standard.

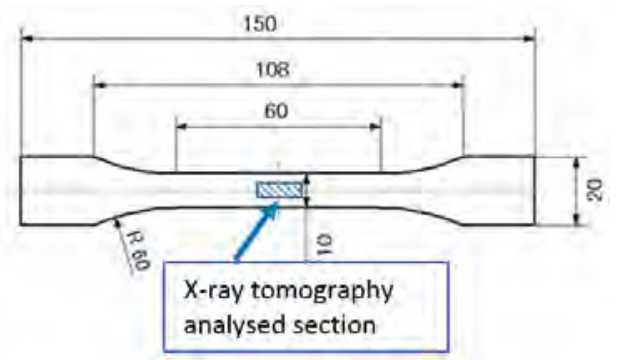

Figure 1 : Fatigue test coupon used (ISO 527B). The plate thickness is $3 \mathrm{~mm}$.

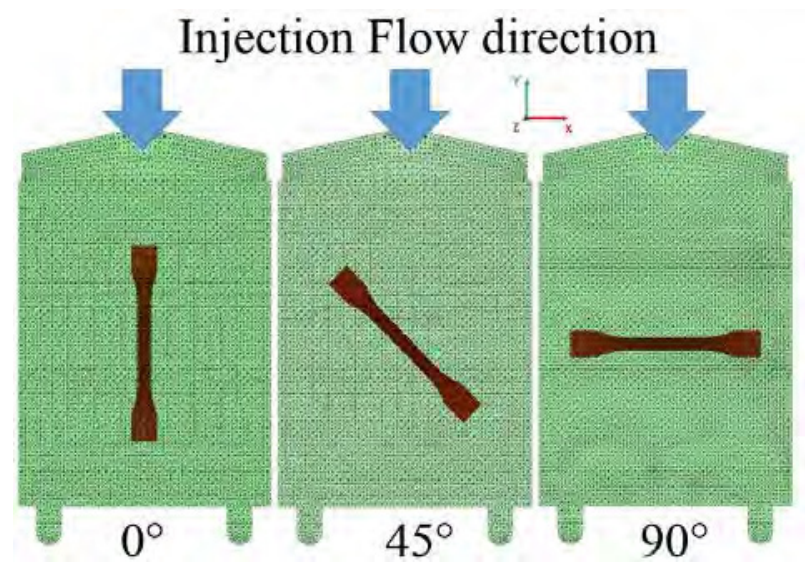

Figure 2: Plate injection and fatigue coupon machining

\subsection{Industrial parts}

The part used in this study is a prototype engine mount. As for the fatigue test coupons, the parts are conditioned using ISO 1110 standard.

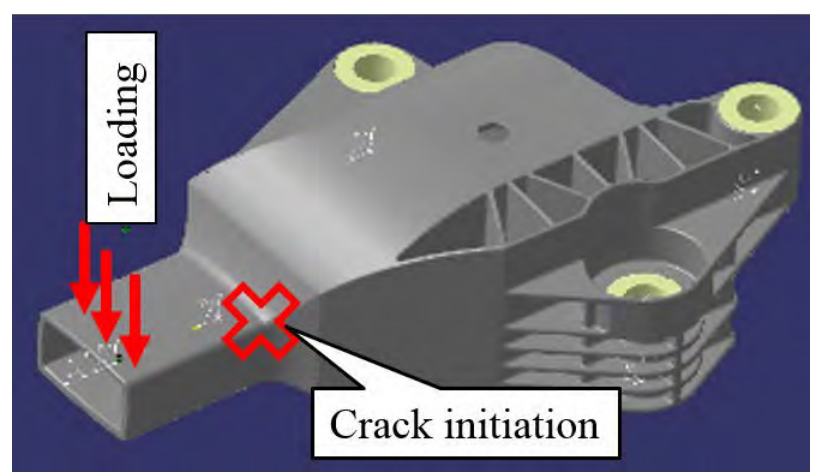

Figure 3: Engine mount studied.

\subsection{Testing}

Fatigue coupon testing was carried out at HBM Prenscia's testing facilities using a compressive tensile machine. To ensure test repeatability and representativeness, the tests were done in a climatic chamber at $23^{\circ} \mathrm{C}$ and $50 \%$ relative humidity. The tests were all conducted in load control using 1 to $3 \mathrm{~Hz}$ test frequency (see part 3.1 for more details). For such long fatigue specimens, undesirable buckling in compression can often occur and a $\mathrm{R}=0.1$ load ratio was therefore used. Further tests, using shorter specimens could be used to investigate compressive loadings. For all tests, the fatigue life is considered as the specimen failure.

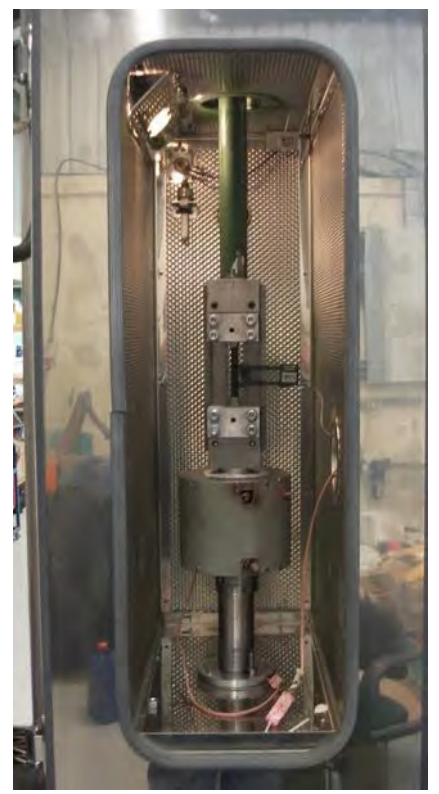

Figure 4: Climatic chamber and fatigue testing rig.

In order to test the engine mount, a specific testing rig was developed (see Figure 5). It allows testing of the part in different orientations. The tests were performed in load control using a load ratio of $\mathrm{R}=0.1$ and a test frequency of $3 \mathrm{~Hz}$. Test were conducted until part failure (severe drop in part rigidity). It must be noted the applied load shown here does not represents loadings occurring in real part situations and was mainly designed to investigate the accuracy of the fatigue criterion. Most of the tests were also monitored using a thermographic camera in order to detect crack initiation (see section 4.2 for more details).

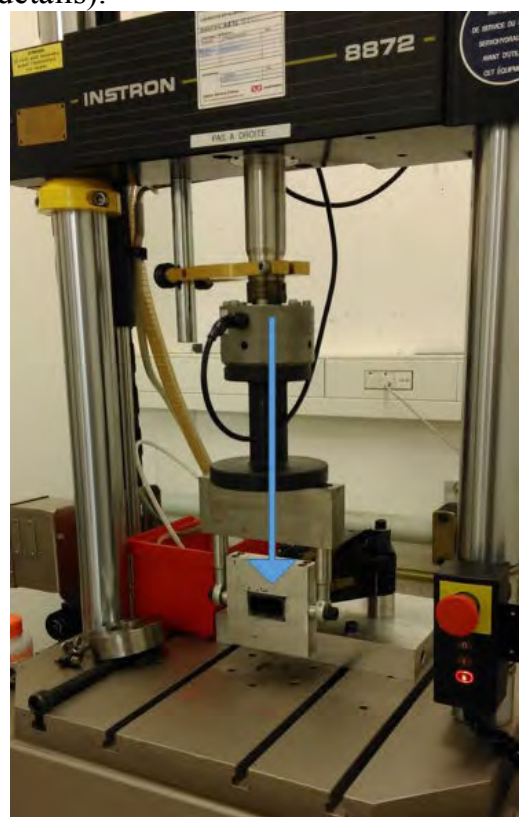

Figure 5: Engine mount testing rig showing loading direction. 


\section{Injection moulding process}

\subsection{Tomography Observations}

In order to better understand the mechanical tests, tomography observation and simulation of the moulding process is of assistance. Samples of material were taken from the central zone of the fatigue specimen (see Figure 1) and analysed by X-Ray tomography. The resulting scan has a $4 \mu \mathrm{m}$ resolution and covers a volume approximately $3 \times 3 \times 3 \mathrm{~mm}$. A slice of the sample analysed can be seen Figure 6. It shows the typical structure of fibre reinforced thermoplastics:

- Surface: while difficult to see on the figure, the surface effect can be seen in 3D, close to the plate surface, the fibres are randomly oriented.

- Skin: this section constitutes the main part of our volume. The fibres are mainly oriented in the injection direction

- Core: this section, of approximately $500 \mu \mathrm{m}$ thick, has fibres perpendicular to the injection direction.

Based on this tomographic data, the fibre tracing algorithm of FEI ${ }^{\circledR}$ Avizo ${ }^{\circledR}$ software and a specific data processing routine was used to study quantitatively the fibre orientation in the specimen. The xx component of the experimentally determined fibre orientation tensor (corresponding to the degree of fibre alignment in the flow direction) is plotted against depth in Figure 7. The $0 \mathrm{~mm}$ depth corresponds to the plate top surface while $3 \mathrm{~mm}$ is the bottom surface. The different sections can clearly be seen (surface, skin and core)

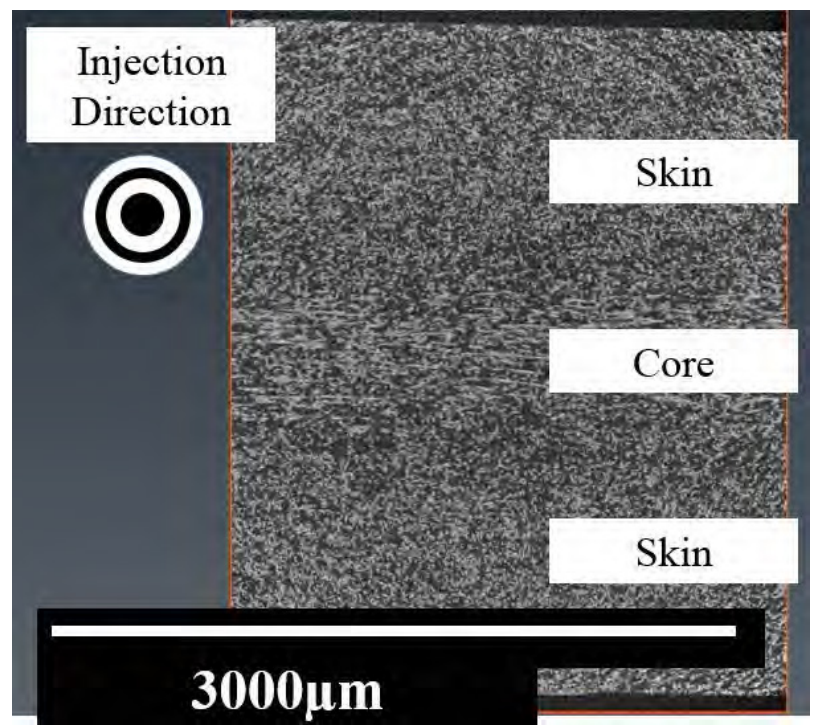

Figure 6: Slice of sample analysed by tomography

\subsection{Injection moulding simulation}

While tomography analysis helps better understand the material microstructure, it remains technically difficult to analyse large volumes, and even more so for parts with complex geometry such as the engine mount studied here, where machining samples can be cumbersome. Therefore, the injection process of the plates and engine mounts was simulated using Autodesk ${ }^{\circledR}$ Moldflow ${ }^{\circledR}$. The moulding simulation generates estimated fibre orientation tensors which for the moulded plates may be compared with the experimental data as shown in Figure 7. The agreement is quite good: the injection simulation displays the same sections as the observed ones, and orientation values appear close. However, the simulation appears to slightly underestimate the degree of fibre orientation in the different layers.

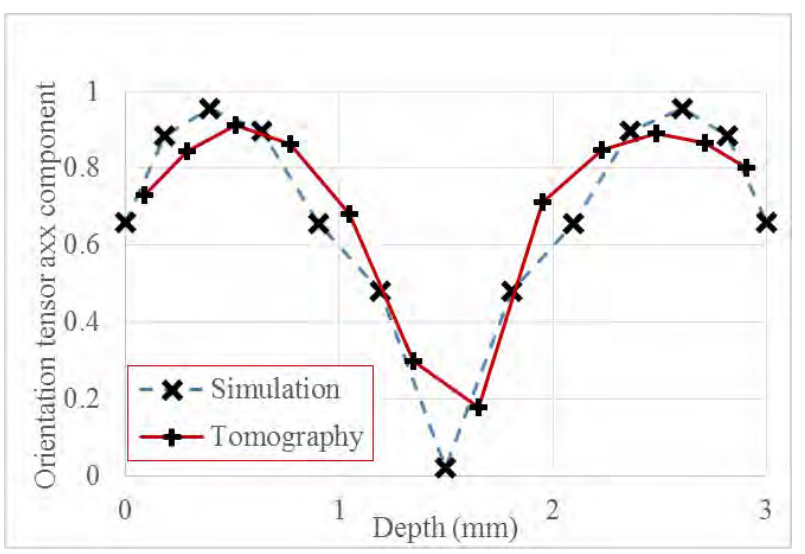

Figure 7: Experimentally observed fibre orientation tensor. The $X$ direction matches the injection direction.

Using the same method, the industrial part injection process was also simulated. The results, shown in Figure 8, can then be used as an input to the integrative mechanical simulation.

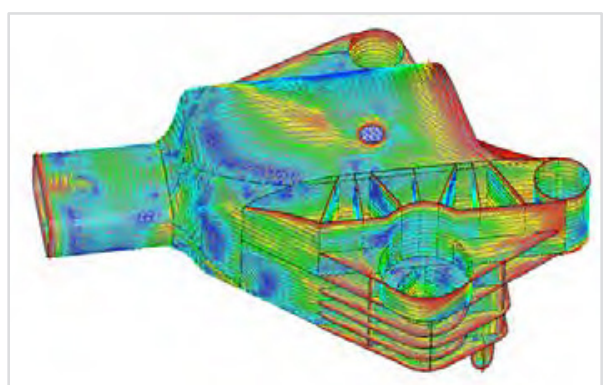

Figure 8: Injection moulding simulation of engine mount showing fibre alignment proportion with local principal direction. A higher value (red) means the fibres are locally aligned, whereas for the lower values (blue), the fibres are locally more randomly oriented.

\section{Experimental results}

\subsection{Specimen heating}

Polyamide based materials are highly sensitive to loading frequency effects [3], even for the lower load levels (i.e. the higher fatigue lives) due at least partly to hysteretic heating. In order to maintain the heating level below a certain threshold, a series of incremental tests were first undertaken to define testing frequency: increasing loads were applied to the specimen and, using 
thermal imaging, the average surface temperature was measured after temperature stabilisation (10000 cycles). These tests were done at $3 \mathrm{~Hz}$ and provide a design of experiment for the fatigue tests. For a given load, if the temperature rise is higher than $5^{\circ} \mathrm{C}$ at $3 \mathrm{~Hz}$, the test frequency is lowered and set to $1 \mathrm{~Hz}$. The results of these tests can be seen in Figure 9 for all three orientations considered.

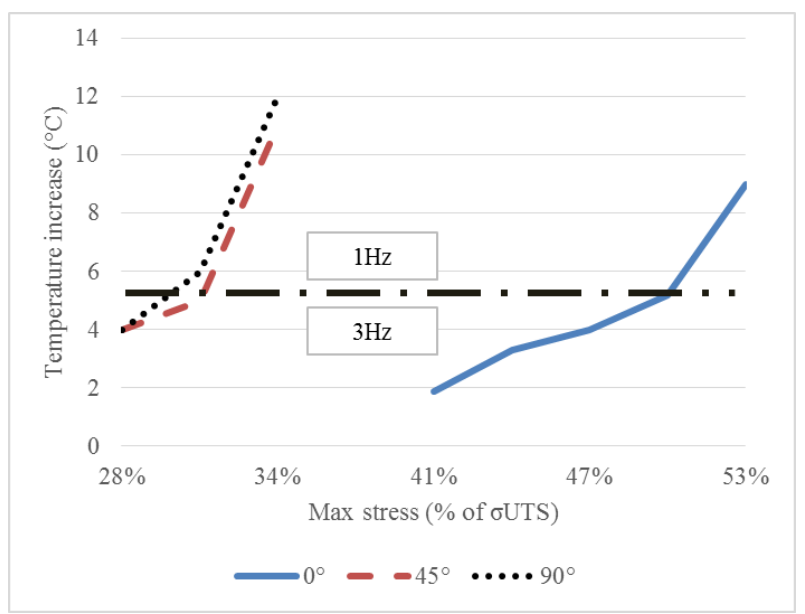

Figure 9: Surface temperature increase for increasing loadings at $3 \mathrm{~Hz}$ of different specimen orientations

\subsection{Fatigue results}

The results of the coupon fatigue tests are presented in Figure 10 for the three different specimen orientations. The $0^{\circ}$ specimen, which is aligned with the injection direction has the best fatigue resistance. Using the microstructure analysis presented in section 3, the influence of fibre orientation appears clearly: when the skin fibres are mainly aligned with the loading direction, the material exhibits the best fatigue resistance. For $45^{\circ}$ specimens, the fatigue resistance lies between that of the $0^{\circ}$ and the $90^{\circ}$ specimens, although closer to $90^{\circ}$ ones.

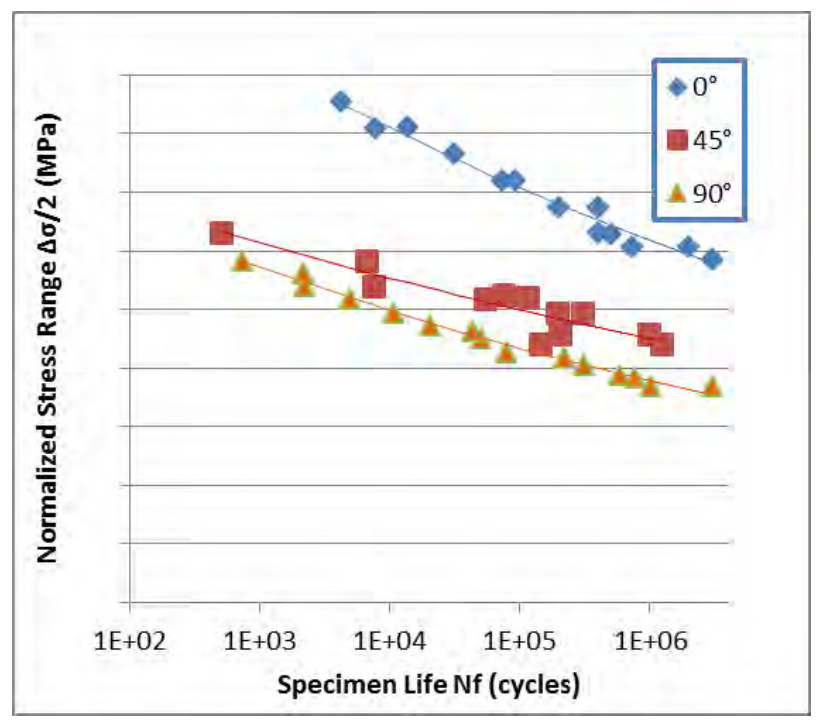

Figure 10: Fatigue test results on PA66GF50.
The results of the fatigue tests on engine mounts are shown in Figure 11, while thermoelasticimetry of the crack initiation location is shown in Figure 12. Rather than standard thermal imaging, thermoelasticimetry allows detection of small variations in stress (see [4] [5]) by synchronizing the measurement signal (here the thermal imaging) with the applied loading signal. Indeed, for a homogenous elastic solid under adiabatic conditions, the temperature variation $\Delta T$ can be linked to the stress tensor $\boldsymbol{\sigma}$ :

$$
\Delta T=-K \cdot T \cdot \operatorname{Tr}(\boldsymbol{\sigma})
$$

Where $T$ is the temperature, $\operatorname{Tr}$ the trace operator and $K$ the thermoelastic constant. (This last constant was not measured so the numerical values of the stress change in Figure 12 are not meaningful.) The interest of this measurement is twofold. Firstly, it allows us to detect hot spots in the structure, which may becompared to the simulation results in section 5.3. Secondly, it allows for a precise detection of crack initiation, which is key to fatigue life assessment.

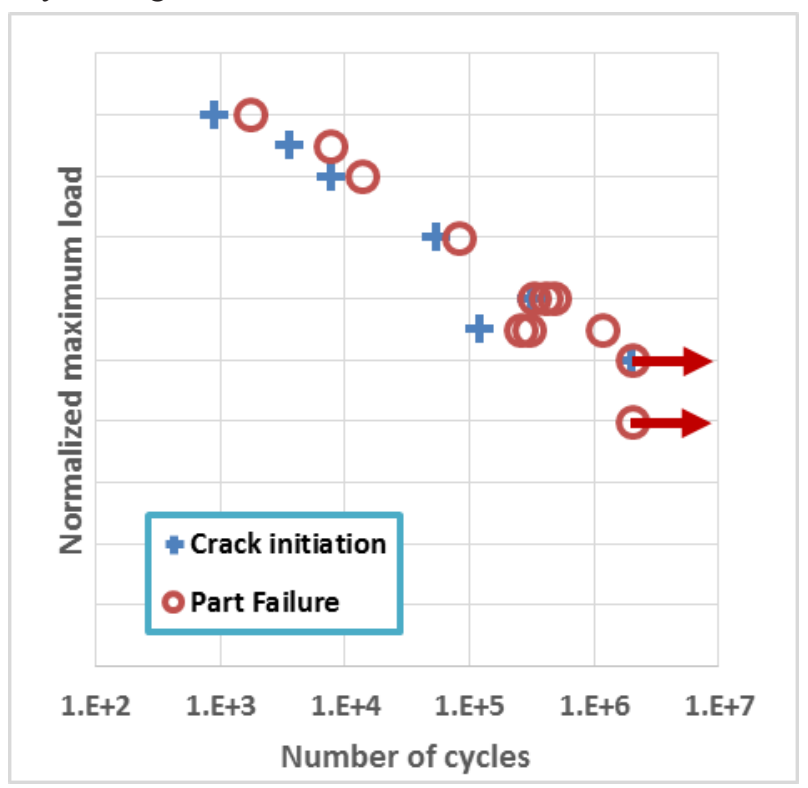

Figure 11: Fatigue test results of engine mount.

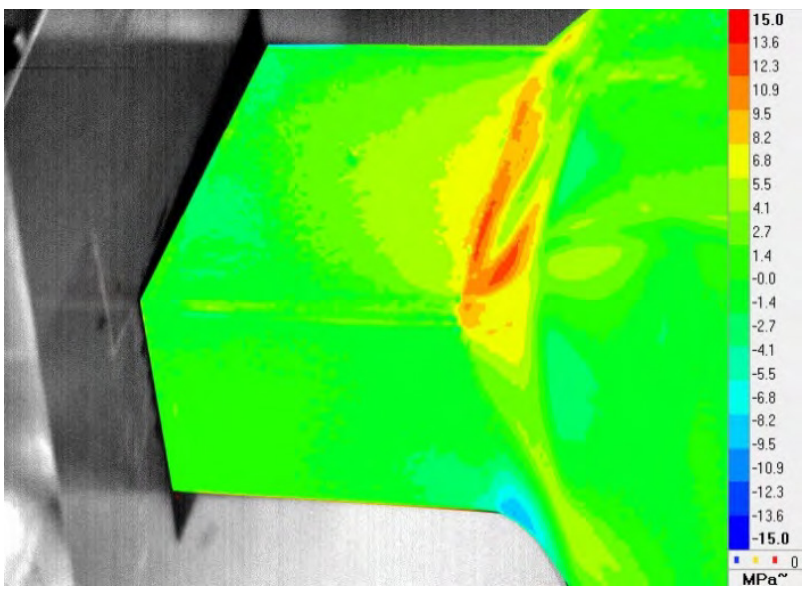

Figure 12: Thermoelasticimetry using thermal imaging camera. 
It must be noted Figure 11 is in logarithmic scale. The crack propagation phase, for this specific configuration, represents between 33 and 54\% (average $44 \%$ ) of the total fatigue life, the part failure being considered as a severe loss of part rigidity. This shows for SFRP parts and long fatigue lives, the crack propagation phases cannot be neglected and can represent half of the total fatigue life.

\section{Mechanical simulation results}

\subsection{Simulation model}

In order to simulate the mechanical tests of fatigue coupons and parts, an integrative simulation method provided by Digimat ${ }^{\circledR}$ and coupled with $A b a q u s\left({ }^{\circledR}\right.$ was used. Based on Mori-Tanaka homogenization models, it allows us to take into account the local microstructure of the material for each layer. All simulations are done using elastic material hypothesis.

The fatigue coupons and the engine mounts were meshed using 2D shell elements. Each element contains 12 layers (where layer 1 is the top surface layer, 6-7 the central layers of the plate, and 12 the bottom surface layer), each defined using the local fibre orientation tensor. Additional microstructural and material data are required: fibre length and aspect ratio, fibre density, Young modulus and Poisson ratio of fibre. The Young modulus of the matrix was not used directly, but rather as a parameter optimised to provide the best fit between test and simulation for the $0^{\circ}$ specimens. Results are shown Table 1 and shows the model is able to correctly simulate the different specimen orientations.

Table 1 : Comparison of experimental and simulated equivalent Young Modulus of tensile tests. The results are normalized by the value of $0^{\circ}$ specimen.

\begin{tabular}{|c|c|c|}
\hline Specimen & Experimental () & Numerical () \\
\hline $0^{\circ}$ & 1.000 & 0.955 \\
\hline $45^{\circ}$ & 0.485 & 0.510 \\
\hline $90^{\circ}$ & 0.465 & 0.497 \\
\hline
\end{tabular}

The optimised material data was then used to simulate the engine mount test. The part stiffness for this loading configuration was found to be very close to the experimental results (less than $10 \%$ error in the part stiffness for the maximum load used in the fatigue tests).

\subsection{Fatigue criteria}

As seen in Figure 10, using equivalent stress (i.e. load divided by specimen section) does not yield satisfactory results. Therefore, a local strain energy density approach is used. The elastic strain energy density $\Delta W_{e}$ is defined by:

$$
\Delta W_{e}=\frac{1}{2} \boldsymbol{\sigma}: \boldsymbol{\varepsilon}_{\boldsymbol{e}}
$$

Where $\boldsymbol{\sigma}$ is the stress tensor and $\boldsymbol{\varepsilon}_{\boldsymbol{e}}$ the elastic strain tensor. The fatigue life $N$ can then be estimated by:

$$
N=A \times \Delta \boldsymbol{W}_{e}^{b}
$$

Where $A$ and $b$ are material parameters. Even though the parameter $A$ can be modified to take into account the stress ratio, it is not detailed in this study, since all tests are done using identical stress ratios. Using HBM Prenscia's nCode DesignLife ${ }^{\circledR}$ software, the elastic strain energy quantity was calculated for the 0,45 and $90^{\circ}$ specimens. Two approaches were then used to calculate the maximum value:

1. The "W max" approach: in this case, the critical value for a specimen is taken as the maximum value all over the elements and all the layers.

2. The "W mean" approach: in this non-local approach, the results are averaged over the specimen thickness (i.e. across all layers). Indeed, each element contains 12 different stress/strain tensors values corresponding to the different layers. For this method, the elastic strain energy density of an element is first calculated by averaging the 12 layers. The critical value of the specimen is then chosen as the maximum value over the specimen.

\subsection{Experimental vs simulation results}

The abilities of the two methods to correlate the coupon test results are shown Figure 13 and Figure 14. These must first be compared to results of stress-life ones (shown in Figure 10): the elastic strain energy density methods minimize differences between the different specimen orientations, making them suitable for complex simulations of industrial parts. Indeed, for these more complex simulations, it is impossible to define an orientation and any fatigue criteria needs unify the test results. For fatigue coupons specimens, no significant differences between the " $\mathrm{W}$ max" and the " $\mathrm{W}$ mean" approach appear.

The two different approaches were then applied to the engine mount parts; see Figure 15 and Figure 16. While results of the "W max" approach appear overconservative, the "W mean" show promising results. Especially for the longer fatigue lives, which are of interest, the results using this last criteria are close to the $/ 10 \times 10$ scatter band. An explanation of the different results between coupons and part simulation could be attributed to the different mechanical loadings: while coupons are loaded in uniaxial tensile loadings, the engine mount undergoes multiaxial and bending loads. Other effects that might be considered are the notch effect (local plasticity and size effect), and mean stress relaxation at stress concentrations when $0<\mathrm{R}<1$ due to accumulating creep strain. 


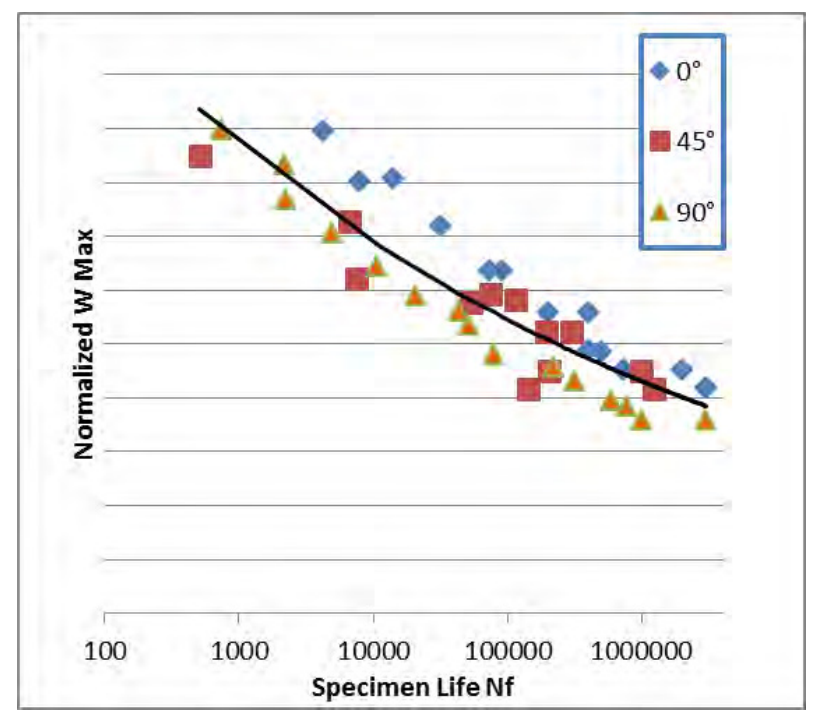

Figure 13: Fatigue results of coupons using the "W max" approach

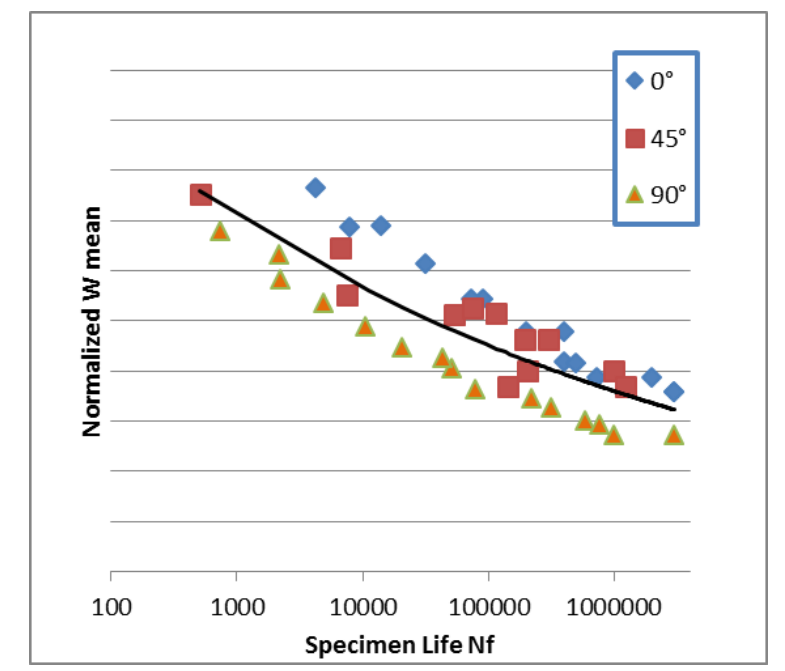

Figure 14: Fatigue results of coupons using the "W mean" approach

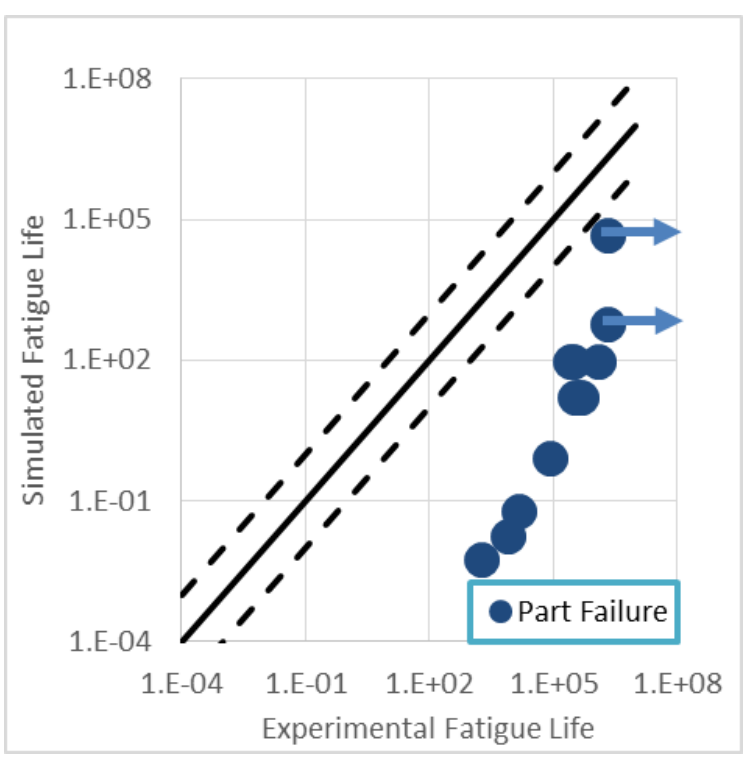

Figure 15: Fatigue results of parts using the "W max" approach

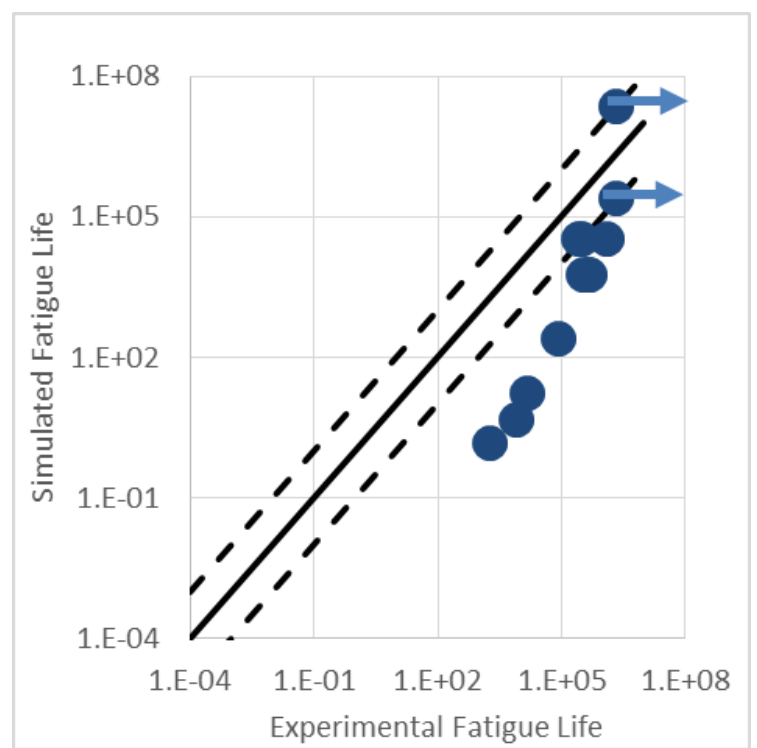

Figure 16: Fatigue results of parts using the "W mean" approach

\section{Conclusions}

An integrative simulation method is used to simulate the fatigue life of a short fibre reinforced plastic (PA66GF50). First, by using X-Ray laboratory tomography and specific image analysis software, the microstructure of the material is investigated, and compared to the results of the moulding injection simulation. Secondly, based on the simulated microstructure, the anisotropic and heterogeneous mechanical behaviour is identified. It shows integrative simulation methods are able to capture and simulate different fibre orientations of specimens. Lastly, using a strain energy based fatigue criterion, the mechanical simulations are used to calibrate the fatigue model based on coupon tests, and assess the life of structural parts. Both local and non-local criteria show promising results.

Further investigation should focus on detailed description of material mechanical behaviour. Indeed, the tests conducted in this study show the material has a strong creep behaviour, especially for the lesser orientated specimens (45 and $90^{\circ}$ specimens), for which the matrix behaviour prevails. Recent studies (see for example [6]) have shown the precise study of this creepfatigue behaviour could help better understand fatigue life assessment.

\section{References}

[1] B. L. Shao-Yun Fu, «Effects of fiber length and fiber orientation distributions on the tensile strength of short-fiber-reinforced polymers,» Composites Science and Technology, 56, Issue 10, 1179-1190, (1996).

[2] H. Rolland, N. Saintier, P. Wilson, J. Merzeau et G. Robert, «In situ X-ray tomography investigation on damage mechanisms in short glass fibre reinforced thermoplastics: Effects of fibre orientation and relative humidity,» Composites Part B: Engineering, 
Volume 109, 170-186, (2017).

[3] S. Mortazavian, A. Fatemi, S. R. Mellot et A.

Khosrovaneh, «Effect of cycling frequency and selfheating on fatigue behavior of reinforced and unreinforced thermoplastic polymers,» Polymer engineering and Science, 55 Issue 10,2355-2367, (2015).

[4] W. J.M.B., «Principles of IR Measurements and Review of Instrumentation Techniques for Therlmoelastic Stress Analysis,» Stress Analysis by Thermoelastic Techniques, (1987).

[5] S. Offermann, E. Merienne et J. L. Beaudoin, «Statistical Method to Evaluate the Amplitude of Cyclical Noisy Signal in IR Thermography,» pp. 4348, (1994).

[6] A. Bernasconi et R. M. Kulin, «Effect of frequency upon fatigue strength of a short glass fiber reinforced polyamide 6: A superposition method based on cyclic creep parameters,» Polymer Composites, 30, issue 2, pp. 154-161, (2009). 